\title{
Fixed Points on Tasković's Upper Transversal Intervally Spaces
}

\author{
IVAN Mitrović
}

\begin{abstract}
The main mathematical object of this paper is Tasković's transversal space. There is a new class of spaces among various kinds of spaces with a possibility of big and significant uses. That includes upper transversal intervally spaces as well as the existence of fixed points and the uniqueness with a large number of specific features and can be a model for various uses in various fields of human creation.
\end{abstract}

\section{INTRODUCTION}

The main mathematical object of this paper is Taskovic's upper transversal space as the furthest space in the hierarchy of geometric spaces. In 1906, in his doctoral thesis, introducing the concept of metric space, Fréchet remarks that instead of triangle inequality a different regularity conditions can also be considered.

Among the various regularity conditions Dj. Kurepa in 1934 replaces triangle inequality with a certain opposite condition defining the corresponding spaces. In 1942 Menger did the same.

Based on these ideas, transversal spaces introduced and defined by Milan Tasković in 1998 have appeared. There are upper and lower transversal spaces and almost all geometric spaces are placed in that scope. Fréchet's, Kurepa's and Menger's spaces specifically fit into that concept of consideration. There is a new class of spaces with big and significant uses. That includes upper and lower transversal intervally spaces.

\section{Transversal upper intervally SPACES}

A fundamental first example of upper transversal spaces for the upper bisection function $g:\left(R_{+}^{0}\right)^{2} \rightarrow R_{+}^{0}:=[0, \infty)$ defined by $g(s, t)=s+t$ is a metric space.

2000 Mathematics Subject Classification. Primary: 47H10, Secondary: 54H25.

Key words and phrases. TCS-convergence, topological spaces, metric spaces, nonlinear conditions of contractions, fixed apex, nonnumerical transverses, completeness, fixed points, forked points, controlling function, Principle of Transpose, transversal spaces. 
In connection with the preceding, the function $\rho: X \times X \rightarrow[a, b] \subset R_{+}^{0}$ for $a<b$ is called an upper (intervally) transverse on $X$ (or upper intervally transversal) iff: $\rho[x, y]=\rho[y, x]$ and if there is an upper (intervally) bisection function $g:[a, b] \times[a, b] \rightarrow[a, b]$ such that

$$
\rho[x, y] \leq \max \{\rho[x, z], \rho[z, y], g(\rho[x, z], \rho[z, y])\}
$$

for all $x, y, z \in X$. A transversal upper intervally space is a set $X$ together with given upper intervally transverse $\rho: X \times X \rightarrow[a, b] \subset R_{+}^{0}$ for $a<b$ on $X$.

In further, a mapping $M: R \rightarrow[a, b] \subset R_{+}^{0}$ for $a<b$ is called an upper (distribution) function if it is nonincreasing, left-continuous with inf $M=a$ and $\sup M=b$. We will denote by $\mathcal{D}$ the set of all upper (distribution) functions.

Distributional upper functions were introduced by M. Tasković in paper [7].

Next two spaces are very interesting examples of transversal upper spaces.

First, an upper statistical space is a pair $(X, \mathcal{R})$, where $X$ is an abstract set and $\mathcal{R}$ is a mapping of $X \times X$ into the set of all upper (distribution) functions $\mathcal{D}$. We shall denote the upper (distribution) function $\mathcal{R}(u, v)$ by $M_{u, v}(x)$ or $M_{u, v}$, whence the symbol $M_{u, v}(x)$ will denote the value of $M_{u, v}$ at $x \in R$. The functions $M_{u, v}$ are assumed to satisfy the following conditions: $M_{u, v}=M_{v, u}, M_{u, v}(c)=b$ for some $c \in R$, and

$$
M_{u, v}(x)=a \text { for } x>c \text { if and only if } u=v,
$$

and if $M_{u, r}(x)=a$ and $M_{r, v}(y)=a$ implies $M_{u, v}(x+y)=a$ for all $u, v, r \in$ $X$ and for all $x, y \in R$.

In view the condition $M_{u, v}(c)=b$, which evidently, implies that $M_{u, v}(x)=$ $b$ for every $x \leq c$. Thus condition (Eq) is equivalent to the statement: $u=v$ if and only if $M_{u, v}(x)=A(x)$, where $A(x)=b$ if $x \leq c$ and $A(x)=a$ if $x>c$.

Obviously, every metric space may be regarded as an upper statistical space of a special kind. One has only to set $M_{u, v}(x)=A(x-d(u, v))$ for every pair of points $(u, v)$ in the metric space $(X, d)$. Also, $M_{u, v}(x)$ may be interpreted as the"measure" that the distance between $u$ and $v$ is less than $x$.

Second example of transversal upper space, an upper intervally space (or Tasković's intervally space from [9]) is a nonempty set $X$ together with the functions $M_{u, v}(x)$ with the following properties: $M_{u, v}=M_{v, u}, M_{u, v}(c)=$ $b$ for some $c \in R,(\mathrm{Eq})$ and if there is a nondecreasing function $f:[a, b] \times$ $[a, b] \rightarrow[a, b]$ with the property $f(t, t) \leq t$ for all $t \in[a, b]$ such that

$$
M_{u, v}(x+y) \leq f\left(M_{u, r}(x), M_{r, v}(y)\right)
$$


for all $u, v, r \in X$ and for all $x, y \geq c$. (Namely, the function $f:[a, b] \times$ $[a, b] \rightarrow[a, b]$ is nondecreasing if $a_{i}, b_{i} \in[a, b]$ and $a_{i} \leq b_{i},(i=1,2)$, implies $\left.f\left(a_{1}, a_{2}\right) \leq f\left(b_{1}, b_{2}\right)\right)$.

We notice, if we chosen an upper bisection (intervally function $g:[a, b] \times$ $[a, b] \rightarrow[a, b]$ such that $g=f$ (from (Nt)), then we immediate obtain that every upper intervally space, for $\rho[u, v]=M_{u, v}$, is a transversal upper intervally space; because in this case from $(\mathrm{Nt})$ the following inequalities hold:

$$
\begin{aligned}
\rho[u, v] & =M_{u, v}(x) \leq f\left(M_{u, r}(x-y), M_{r, v}(y)\right):= \\
& :=g(\rho[u, r], \rho[r, v]) \leq \max \{\rho[u, r], \rho[r, v], g(\rho[u, r], \rho[r, v])\} .
\end{aligned}
$$

On the other hand, if: $M_{u, v}=M_{v, u}, M_{u, v}(c)=b$ for some $c \in R,(\mathrm{Eq})$, and if there is a function $\Psi:[a, b] \times[a, b] \rightarrow[a, b]$ such that

$$
M_{u, v}(x) \leq \Psi\left(M_{u, r}(x), M_{r, v}(x)\right)
$$

for all $u, v, r \in X$ and for every $x \geq c$, then it is an example of transversal upper intervally space also.

A mapping $\Delta:[0,1] \times[0,1] \rightarrow[0,1]$ is a $\Delta$-norm if it satisfies: $\Delta(a, 1)=$ $a, \Delta(0,0)=0, \Delta(a, b)=\Delta(b, a), \Delta(c, d) \geq \Delta(a, b)$ for $c \geq a, d \geq b$ and $\Delta(\Delta(a, b), c)=\Delta(a, \Delta(b, c))$.

Let $\mathcal{B}$ denote the set of all $\Delta$-norms, partially ordered by $\Delta_{1} \leq \Delta_{2}$ if and only if $\Delta_{1}(a, b) \leq \Delta_{2}(a, b)$ for all $a, b \in[0,1]$ and $\Delta_{1}, \Delta_{2} \in \mathcal{B}$.

In connection with this, an upper probabilistic $M T$-space is a triplet $(X, \mathcal{R}, \Delta)$, where $(X, \mathcal{R})$ is an upper statistical space and $f \in \mathcal{B}$ satisfies the preceding triangle inequality $(\mathrm{Nt})$.

A very characteristic example, for further work, of the transversal upper intervally spaces is the following space in the following form.

A transversal upper intervally $T$-space is a pair $(X, \rho)$, where $X$ is a transversal upper intervally space and where the upper (intervally) transverse $\rho[u, v]=M_{u, v}(x)$ satisfying: $M_{u, v}=M_{v, u}, M_{u, v}(c)=b$ for some $c \in R$ and $(\mathrm{Eq})$.

These spaces were introduced by M. Tasković in paper [7].

\section{MAIN RESUlT}

Theorem 1. Let $(X, \rho)$ be a complete transversal upper intervally $T$-space, where upper transveral is $\rho[u, v]=M_{u, v}(x)$ and upper bisection function $g:[a, b]^{2} \rightarrow[a, b]$ is nondecreasing such that $g(t, t) \leq t$. Then the following condition is sufficient for the existence of a unique fixed point of mapping $T: X \rightarrow X$ in the form of:

(i) (Case $a>0$ of $[a, b]$ ). There is a nondecreasaing function $\varphi$ : $[c,+\infty) \rightarrow[c,+\infty):=I_{c}$ for some fixed $c \in R$ with the properties that

$$
(A s) \lim _{n \rightarrow \infty} \varphi^{n}(t)=+\infty \quad \text { for every } t>c
$$


such that for all $u, v \in X$ and for every $x>c$ inequality is true in the form of

$$
\begin{array}{r}
M_{T u, T v}(x) \leq \max \left\{M_{u, v}(\varphi(x)), \frac{M_{u, T u}(\varphi(x)) M_{u, T v}(\varphi(x))}{M_{u, v}(\varphi(x))},\right. \\
\left.\frac{M_{u, T v}(\varphi(x)) M_{v, T u}(\varphi(x))}{a}\right\}
\end{array}
$$

Proof. Proof of existence of a fixed point, let $u \in X$ be arbitrary point and let us define the sequence of iterations $u_{n}=T^{n}(u)$ for $n \in N \cup\{0\}$. Let us show that the sequence $\left\{u_{n}\right\}_{n \in N \cup\{0\}}$ is fundamental Cauchy's sequence from $X$. Then it is true for $t>c$ and $m>n(m, n \in N)$ according to $(A)$ that

$$
M_{u_{n}, u_{m}}(t) \leq \max \left\{M_{u_{n}, u_{n+1}}(t), \ldots, M_{u_{m-1}, u_{m}}(t)\right\} .
$$

Since $T u_{n}=u_{n+1}$ and $T u_{n-1}=u_{n}$ by the change in (1) we get for every $t>c$ the following estimate

$$
\begin{aligned}
M_{u_{n}, u_{n+1}}(t)= & M_{T u_{n-1}, T u_{n}}(t) \leq \\
\leq & \max \left\{M_{u_{n-1}, u_{n}}(\varphi(t)),\right. \\
& \frac{M_{u_{n-1}, T u_{n-1}}(\varphi(t)) M_{u_{n-1}, T u_{n}}(\varphi(t))}{M_{u_{n-1}, u_{n}}(\varphi(t))}, \\
& \left.\frac{M_{u_{n-1}, T u_{n}}(\varphi(t)) M_{u_{n}, T u_{n-1}}(\varphi(t))}{a}\right\},
\end{aligned}
$$

i.e.,

$$
\begin{aligned}
M_{u_{n}, u_{n+1}}(t)= & M_{T u_{n-1}, u_{n}}(t) \leq \\
\leq & \max \left\{M_{u_{n-1}, u_{n}}(\varphi(t)),\right. \\
& \frac{M_{u_{n-1}, u_{n}}(\varphi(t)) M_{u_{0}, u_{n+1}}(\varphi(t))}{M_{u_{n-1}, u_{n}}(\varphi(t))}, \\
& \left.\frac{M_{u_{n-1}, u_{n+1}}(\varphi(t)) \overbrace{M_{u_{n}, u_{n}}(\varphi(t))}^{a}}{a}\right\}
\end{aligned}
$$

Since $M_{u, v}(\varphi(t))=a$ if and only if $u=v$, then from the preceding facts

$$
\begin{aligned}
M_{u_{n}, u_{n+1}}(t) \leq \max \{ & M_{u_{n-1}, u_{n}}(\varphi(t)), M_{u_{n-1}, u_{n+1}}(\varphi(t)), \\
& \left.M_{u_{n-1}, u_{n+1}}(\varphi(t))\right\} \\
\leq \max \{ & \left.M_{u_{n-1}, u_{n}}(\varphi(t)), M_{u_{n}, u_{n+1}}(\varphi(t))\right\}
\end{aligned}
$$

and therefore

$$
M_{u_{n}, u_{n+1}}(\varphi(t)) \leq \max \left\{M_{u_{n-1}, u_{n}}\left(\varphi^{2}(t)\right), M_{u_{n}, u_{n+1}}\left(\varphi^{2}(t)\right)\right\} .
$$


According to the estimates (3) and (4) we inductively get for every $k \in N$ that the following inequality is true

$$
M_{u_{n}, u_{n+1}}(\varphi(t)) \leq \max \left\{M_{u_{n-1}, u_{n}}(\varphi(t)), M_{u_{n}, u_{n+1}}\left(\varphi^{k}(t)\right)\right\}
$$

i.e. when $k \rightarrow \infty$, we get $M_{u_{n}, u_{n+1}}(t) \leq M_{u_{n-1}, u_{n}}(\varphi(t))$ for every $n \in N$, i.e. we get

$$
M_{u_{n}, u_{n+1}}(t) \leq M_{u_{0}, u_{1}}\left(\varphi^{n}(t)\right)
$$

for every $n \in N$. Therefore, using this fact and previous inequality (2) we get inequality and equality

(7)

$$
M_{u_{n}, u_{m}}(t) \leq \max \left\{M_{u_{0}, u_{1}}\left(\varphi^{n}(t)\right), \ldots, M_{u_{0}, u_{1}}\left(\varphi^{m-1}(t)\right)\right\}=M_{u_{0}, u_{1}}\left(\varphi^{n}(t)\right),
$$

therefore, we get that $\left\{u_{n}\right\}_{n \in N}$ is one Cachy's or fundamental sequence from $X$. It means (because of the space $X$ completeness) that there is point $p \in X$ and so $u_{n} \rightarrow p$, i.e. $T^{n}(u) \rightarrow p$.

Since $(E q)$ and the fact $u \neq v$ according to previous conclusions, we get

$$
M_{u, v}(\varphi(t))<M_{u, v}(t) \text { for some } t>c .
$$

From inequality (1) we get

$$
\begin{array}{r}
M_{T\left(u_{n}\right), T(p)} \leq \max \left\{M_{u_{n}, p}(\varphi(t)), \frac{M_{u_{n}, u_{n+1}}(\varphi(t)) M_{u_{n}, T(p)}(\varphi(t))}{M_{u_{n}, p}(\varphi(t))},\right. \\
\left.\frac{M_{u_{n}, T(p)}(\varphi(t)) M_{p, u_{n+1}}(\varphi(t))}{a}\right\}
\end{array}
$$

for every $t>c$ and every $n \in N$. Therefore as $T\left(u_{n}\right)=u_{n+1}$ for $n \rightarrow \infty$ according to (9), we get $M_{p, T p}(t) \leq M_{p, T p}(\varphi(t))$ for every $t>c$. This means according to (8) that $T p=p$.

Let us prove uniqueness of point $p \in X$. Let $T p=p \neq q=T q$ by changing in (1)

$$
\begin{aligned}
M_{p, q}(x) \leq \max \left\{M_{p, q}(\varphi(x)),\right. & \frac{M_{p, p}(\varphi(x)) M_{p, q}(\varphi(x))}{M_{p, q}(\varphi(x))}, \\
& \left.\frac{M_{p, q}(\varphi(x)), M_{q, p}(\varphi(x))}{a}\right\} .
\end{aligned}
$$

Since $M_{p, q}(\varphi(x))=a$ is equivalent to $p=q$ we have

$$
M_{p, q}(x) \leq \max \left\{M_{p, q}(\varphi(x)), a, \frac{M_{p, q}^{2}(\varphi(x))}{a}\right\} .
$$

from previous we have by induction $M_{p, q}(x) \leq M_{p, q}(\varphi(x)) \leq M_{p, q}\left(\varphi^{2}(x)\right) \leq$ $\cdots \leq M_{p, q}\left(\varphi^{n}(x)\right)$ which means that, considering the fact $M_{p, q}\left(\varphi^{n}(x)\right) \rightarrow a$ for $n \rightarrow \infty$, we get by changing in(11)

$$
M_{p, q}(x) \leq \max \left\{a, a, \frac{a^{2}}{a}\right\}=a
$$


i.e. $M_{p, q}(x)=a$ is equivalent to $p=q$ we get a contradiction to the our assumption, which means that fixed point of mapping $T$ is unique. From this, the proof of the theorem is complete.

Remarks. We notice that in the case $a=0$ of $[a, b]$ with the conditions of the previous theorem, the following case will be considered as a sufficiently for the existence of at least one fixed point of the mapping $T: X \rightarrow X$ :

There is a nondecreasing function $\varphi: I_{c} \rightarrow I_{c}$ for some fixed $c \in R$ with properties (As) such that for all $u, v \in X(u \neq v)$ and for every $x>c$ inequality is true in the form of

$$
\begin{aligned}
M_{T u, T v}(x) & \leq \frac{M_{u, T u}(\varphi(x)) M_{v, T v}(\varphi(x))}{M_{u, v}(\varphi(x))}+ \\
& +r(u, v) \frac{M_{u, T v}(\varphi(x)) M_{v, T u}(\varphi(x))}{M_{u, v}(\varphi(x))}
\end{aligned}
$$

where $(u, v) \mapsto r(u, v)$ is an arbitrary nonnegative bounded function. If $r(u, v) \leq 1$, then $T$ has a unique fixed point. Also, without of completeness of space $X, T$ has at least one fixed point: by Tasković [10].

Proof. For proof of fixed point existence, let $u \in X$ be arbitrary point and let us define the sequence of iterations $u_{n}=T^{n}(u)$ for $n \in N \cup\{0\}$. Let us show that the sequence $\left\{u_{n}\right\}_{n \in N \cup\{0\}}$ is fundamental Cauchy's sequence from $X$. Then it is true (2) for $t>c$ and $m>n(m, n \in N)$ according to (As).

Since $T u_{n}=u_{n+1}$ and $T u_{n-1}=u_{n}$ by the change in (12) we get for every $t>c$ the following estimate

$$
\begin{aligned}
M_{u_{n}, u_{n+1}}(t)= & M_{T u_{n-1}, T u_{n}}(t) \leq \\
\leq & \frac{M_{u_{n-1}, T u_{n}-1}(\varphi(t)) M_{u_{n}, T u_{n}}(\varphi(t))}{M_{u_{n-1}, u_{n}}(\varphi(t))}+ \\
& +r\left(u_{n-1}, u_{n}\right) \frac{M_{u_{n-1}, T u_{n}}(\varphi(t)) M_{u_{n}, T u_{n-1}}(\varphi(t))}{M_{u_{n-1}, u_{n}}(\varphi(t))} \\
M_{u_{n}, u_{n+1}}(t) \leq & \frac{M_{u_{n-1}, u_{n}}(\varphi(t)) M_{u_{n}, u_{n+1}}(\varphi(t))}{M_{u_{n-1}, u_{n}}(\varphi(t))}+ \\
& +r\left(u_{n-1}, u_{n}\right) \frac{M_{u_{n-1}, u_{n+1}}(\varphi(t)) M_{u_{n}, u_{n}}(\varphi(t))}{M_{u_{n-1}, u_{n}}(\varphi(t))} .
\end{aligned}
$$

Since $M_{u, v}(\varphi(t))=a$ if and only if $u=v$, then

$$
M_{u_{n}, u_{n+1}}(t) \leq M_{u_{n}, u_{n+1}}(\varphi(t))+r\left(u_{n-1}, u_{n}\right) \frac{M_{u_{n-1}, u_{n+1}}(\varphi(t)) \cdot a}{M_{u_{n-1}, u_{n}}(\varphi(t))}
$$

for $a=0$ with the condition that $0 \leq r\left(u_{n-1}, u_{n}\right) \leq M<\infty$ we get

$$
M_{u_{n}, u_{n+1}}(t) \leq M_{u_{n}, u_{n+1}}(\varphi(t)) .
$$


For every $n \in N$, i.e., by induction we get

$$
M_{u_{n}, u_{n+1}}(t) \leq M_{u_{0}, u_{1}}\left(\varphi^{n}(t)\right) .
$$

Therefore, applying this fact and earlier inequality (2) and inequality (7) we get that $\left\{u_{n}\right\}_{n \in N}$ is one Cauchy's or fundamental sequence from $X$. It means (because of the space $X$ completeness) that there is point $p \in X$ and so $u_{n} \rightarrow p$, i.e., $T^{n}(u) \rightarrow p$.

Since (Eq) and the fact $u \neq v$ according to previous conclusions, we get

$$
M_{u, v}(\varphi(t))<M_{u, v}(t) \text { for some } t>c .
$$

Therefore, since $T\left(u_{n}\right)=u_{n+1}$ for $n \rightarrow \infty$, condition (15) and (16) mean for every $t>c$ that $T p=p$.

Let us prove uniqueness.

Let us assume that $p$ is not a fixed point but there is such $q$ that $p \neq q$.

Then (i) $T p=p \neq q=T q$ by the change in (12) we get

$$
\begin{aligned}
& M_{p, q}(x) \leq \frac{M_{p, p}(\varphi(x)) M_{q, q}(\varphi(x))}{M_{p, q}(\varphi(x))}+ \\
& +r(p, q) \frac{M_{p, q}(\varphi(x)), M_{q, p}(\varphi(x))}{M_{p, q}(\varphi(x))} .
\end{aligned}
$$

Since for $r(p, q) \leq 1$ is $M_{p, q}(x) \leq M_{p, q}(\varphi(x))$ by induction we get the following inequalities

$$
M_{p, q}(x) \leq M_{p, q}(\varphi(x)) \leq \cdots \leq M_{p, q}\left(\varphi^{n}(x)\right)
$$

and when $n \rightarrow \infty$, then $M_{p, q}\left(\varphi^{n}(x)\right) \rightarrow a$. Since $M_{p, p}(\varphi(x))=a$ by the change in (17) we get

$$
M_{p, q}(x) \leq \frac{a \cdot a}{M_{p, q}(\varphi(x))}+r(p, q) M_{p, q}(\varphi(x)),
$$

i.e., $p=q$. This is contradicts. The proof is now complete.

\section{REFERENCES}

[1] Maurice Fréchet, La notion d'écart et le calcul fonctionnel, C.R. Acad. Sci., Paris, 140(1905) 772-774.

[2] Maurice Fréchet, Sur quelques points du calcul fonctionnel, Thése, Paris 1905; Renidiconti Circolo Mat. Palermo, 22(1906), 1-74.

[3] Djuro R. Kurepa, Tableaux ramifiés, d'ensembles. Espaces pseudo-distanciés, C. R. Acad. Sci. Paris, 198(1934), 1563-1565.

[4] Djuro R. Kurepa, Ensembles ordonnés et ramifiés, Thése, Paris 1935, 1-138; Publ. Inst. Math. Belgrade, 4(1935), 1-138.

[5] Karl Menger, Untersuchungen über allgemeine Metrik, Math. Annalen, 100(1928), 75-163.

[6] Karl Menger, Statistical metric, Proc. N. Acad. Sci., USA, 28(1942), 535-537.

[7] M.R. Tasković, Transversal intervally spaces, Math. Moravica 7(2003), 91-106. 
[8] M.R. Tasković, Fixed points on transversal probabilistic spaces, Math. Moravica 3(1999), 77-82.

[9] M.R. Tasković, Transversal spaces, Math. Moravica 2(1998), 133-142.

[10] M.R. Tasković, Theory of transversal point, spaces and forks, Monographs of a new mathematical theory, Beograd (2005) 1054 pages (In Serbian), VIZ - Beograd, English Summary, 1001-1022.

IVAN Mitrović

ČOČETOVA 20

35000 JAGODINA

SERBia

E-mail address: imitrovic@open.telekom.rs 